\title{
The Best Route Is Not Always the Easiest One: Spatial References in Heuristics of Route Choice
}

\author{
Wen Wen ${ }^{1}$, Hideaki Kawabata ${ }^{2}$ \\ ${ }^{1}$ Advanced Research Centers, Keio University, Tokyo, Japan \\ ${ }^{2}$ Department of Psychology, Faculty of Letters, Keio University, Tokyo, Japan \\ Email:kawabata@flet.keio.ac.jp
}

Received July $1^{\text {st }}, 2013$; revised August $3^{\text {rd }}, 2013$; accepted August $27^{\text {th }}, 2013$

\begin{abstract}
Copyright (C) 2013 Wen Wen, Hideaki Kawabata. This is an open access article distributed under the Creative Commons Attribution License, which permits unrestricted use, distribution, and reproduction in any medium, provided the original work is properly cited.
\end{abstract}

\begin{abstract}
In the present study, we discovered a relationship between down-going and up-going route preferences and selection of spatial reference. The participants were asked to choose between a down-going route and an up-going route on a simplified map. When they were asked to select the better route (Experiment 1), they preferred the down-going route, although the two routes were the same shape and distance. However, when the participants were asked to select the route that seemed easier to remember and find, they favored up-going routes (Experiment 2). We suggested that the contrary route preferences were caused by different selections of spatial references. That is, the first instruction directed participants' attention to the configurational layout of the maps (i.e., promoted the allocentric reference) and induced the down-going route preference, whereas the later instruction promoted egocentric navigating strategies and induced the up-going route preferences. Furthermore, we asked the participants to learn a down-going and an up-going route, then examined their wayfinding and spatial memory performance (Experiment 3 ). The participants found the goals more quickly when up-going routes were used, but remembered the locations of landmarks more accurately when down-going routes were used.
\end{abstract}

Keywords: Route Choice; Spatial Reference; Allocentric Reference; Egocentric Reference; Geographic Reference; Sense of Direction

\section{Introduction}

When you are traveling in a novel city, you probably need to check maps and plan a route from the nearest subway station to your hotel. Most often, there is more than one route choice. For example, there may be several stations nearby, so you need to decide which one to use and choose the best route. However, the definition of the "best route" is ambiguous. It might refer to the shortest one, the simplest one, or the one easiest to find. According to previous findings on route choice, people do not always choose the shortest route (Bailenson, Shum, \& Uttal, 1998, 2000). Instead, their route-finding decisions depend on the use of general heuristics (Bailenson et al., 1998; Brunyé et al., 2012; Brunyé, Mahoney, Gardony, \& Taylor, 2010; Yang \& Schwaninger, 2011). For instance, people tend to select the street most in line with the target (Hochmair \& Karlsson, 2005), or prefer the extreme routes over the middle ones although all the routes were the same length and required the same number of turns (Christenfeld, 1995). Furthermore, the routes with straight initial segments are preferred even though the routes may not be the shortest in distance (Bailenson et al., 1998, 2000).

In addition to the studies mentioned above, Brunyé et al. $(2010,2012)$ recently reported a novel heuristics of southern route preference, which causes participants to preferentially choose a south-going route over a north-going route during map-based route planning. Brunyé et al. (2010) attributed this heuristic to the misperception of increases associated with the direction, north (i.e., north is up). In line with this heuristic, participants rated a northern route as one that burned more calories and took more time to complete than a southern route. In addition, Brunyé et al. (2012) found that large-scale regional characteristics did not affect the southern route heuristic, and speculated that a north-going route may be associated with a vertically upward direction, which is more physically demanding relative to the downward direction. However, in the studies by Brunyé et al. (2010, 2012), the south-going routes appeared both south-going and down-going routes, because the north was always shown as up in all the maps used in their experiments. Therefore, it is possible that the so-called southern route preference heuristic is not actually related to geographic reference (i.e., north-south); instead, it may be due to an egocentric reference (i.e., up-down). Furthermore, in the Brunyé et al. studies (2010, 2012: p. 301), the best route was explained as "the one that was shorter and/or faster," although there were probably individual differences in the comprehension of the "best route."

In the present study, we re-examined the heuristics of southern route preference proposed by Brunyé et al. $(2010,2012)$. We separated geographic and egocentric reference, examined the effect of different instructions on route preference, and tested behavioral facilitations of a down-going route and an up-going route. First, we asked people to choose the "better route" from two different origins, which were above or below the same destination, and the "better route" from the same ori- 
gin to two different destinations, which were above or below the origin, to test whether the southern route preference heuristic also refers to the south-toward (or down-toward) route (i.e., a route with a northern origin and a southern destination). Second, we added rotated compasses to the maps and changed the instruction to "choose the route easier to go," to examine the effects of references and instructions on route preference. Third, we asked the participants to remember maps of up-going or down-going routes, and measured their wayfinding and spatial memory performance.

\section{Experiment 1}

In the Brunyé et al. studies (2010, 2012), the heuristic was called the south-going route preference, in which the southern routes went toward south at first but turned to north later; even the destinations were to either the east or the west of the origins (i.e., the start locations). Here, we first used conditions with two origins or two destinations to determine whether this heuristic also includes a preference for a south-toward route. In the two-origin condition, imagine that there were two different railway stations near a hotel (the distance between the hotel and the different stations was the same). In the two-destination condition, imagine that there were two different hotels, one to the north of the station and another to the south (the hotels were equal on all other factors). In both conditions, the participants were required to select the better route from one of the two origins or to one of the two destinations.

\section{Method}

\section{Participants}

Sixty-eight participants ( 33 female, mean age $=24.6$ years, 4 left-handers) completed the route choice questionnaire. Of the participants, 43 completed paper questionnaires and 25 completed the same questionnaire via an online Google form.

\section{Materials and Procedure}

Two types of maps were used. One map type contained two origins and one destination (two-start maps, upper panels in Figure 1, (A1)-(A5)), and the other map type contained one origin and two destinations (two-goal map, lower panels in Figure 1, (B1)-(B5)). For each two-start map, in the up-down condition, there were two origins (i.e., start locations), presented as small circles at the top and the bottom, and one destination, presented as a star in the center. In the left-right condition, the two origins were presented at the left side and the right side. The straight line distances between the origins and the destinations were the same in all the maps. For each map, two routes started from the origins and ended at the destination. The upper and the lower route had the same shape (the routes were rotated $180^{\circ}$ from each other). Black arrows were presented near the origins, showing the route directions. The routes were curved, at oblique angles, at right angles, or in a straight line (two maps for each route shape). Furthermore, the routes in half of the maps started toward the destination (the start-toward-goal condition), while the others started toward the direction opposite the destination (the start-against-goal condition) (e.g., Figure 1, A5). All the left-right maps were modified from the updown maps. In fact, to obtain the left-right maps, the up-down maps were rotated $90^{\circ}$. For each two-goal map, there was an origin in the center and two destinations beside the origin. The two-goal maps were modified from the two-start maps. In fact, to obtain the two-goal maps, the positions of the origins and destinations of the two-start maps were exchanged. Sixteen

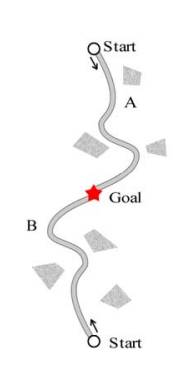

(AI)

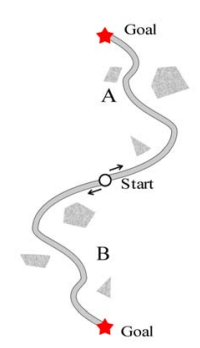

(B1)

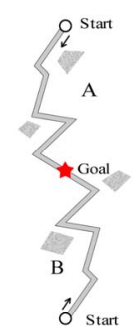

(A2)

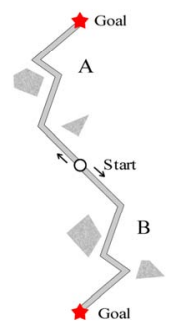

(B2)

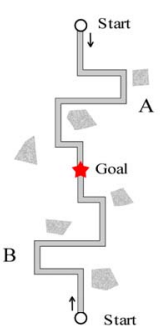

(A3)

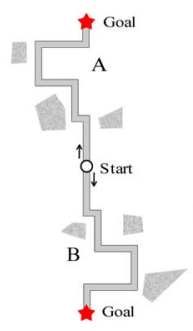

(B3)

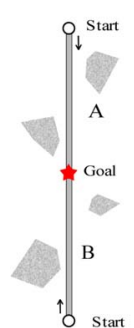

(A4)

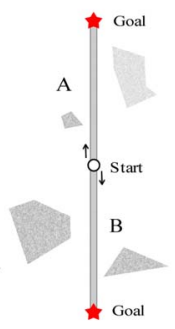

(B4)

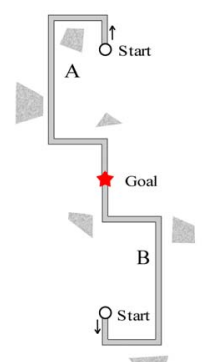

(A5)

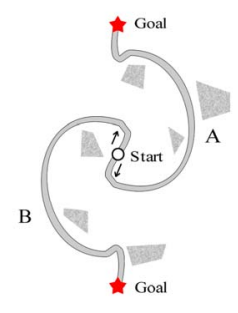

(B5)

Figure 1.

Examples of the maps used in Experiment 1. (A1)-(A5) are two-start maps, which contained two origins and one destination. (B1)-(B5) are two-goal maps, which contained one origin and two destinations. Origins described as "Start" are presented as white circles, and destinations described as "Goal" are presented as red-filled stars. The routes were printed in gray color with black borders. Black arrows near the starts showed the directions of the routes. Gray polygons, located randomly along the routes, served as landmarks. 
two-start maps and fourteen two-goal maps (two-goal maps containing a straight route with opposite start directions were not used) were included in the questionnaire.

The two-start maps and the two-goal maps were grouped in different parts of the questionnaire. The participants started with the two-start maps and were asked to select the better route to the goal for each map. Next, for each two-goal map, they selected the route they preferred to use. Maps were printed in random order. The participants were asked to make intuitive decisions and not to change their prior choices.

\section{Results}

For all up-down maps, the percentage of down-going routes selected is given in Table 1. Similarly, for all left-right maps, the percentage of right-going routes selected is also given in Table 1. The selections were compared to chance level (50\%) after the application of angular transformations. For the twostart maps, the selections of the down-going routes were significantly greater than chance level for both the start-towardgoal condition and the start-against-goal condition $(t(67)=3.35$, $p<.01 ; t(67)=3.06, p<.01$, respectively). Right-going routes were selected at chance level for both start direction conditions (start-toward-goal condition: $t(67)=0.96$, n.s.; start-againstgoal condition: $t(67)=1.58$, n.s.). In contrast, for the two-goal maps, the selections of down-going routes were significantly less than chance level for the start-toward-goal condition ( $t(67)$ $=-3.46, p<.01$ ), whereas the selections of down-going routes in the start-against-goal condition, the selections of right-going routes in the start-toward-goal and start-against-goal conditions were all at chance level $(t(67)=1.42$, n.s.; $t(67)=1.96$, n.s.; $t(67)=-0.16$, n.s., respectively). Moreover, there were significantly more selections of down-going routes in the startagainst-goal condition than in the start-toward-goal condition $(t(67)=4.06, p<.01)$.

\section{Discussion}

Experiment 1 demonstrated that the participants preferred the down-going route (to one of the two destinations) when they were asked to choose the "better one," although a definition of the "better route" was not provided. However, they preferred an up-going route (from one of the two origins) when they were asked to choose "the one they preferred to use." The contrary route direction preferences were quite interesting, indicating that the "better route" may not equal the one people actually considering using. A possible explanation for the results was that people probably prefer the routes on the upper side of the map, since both down-going routes in the two-start condition and up-going routes in the two-goal condition were located in the upper half of the maps. However, this explanation is not consistent with the Brunyé et al. $(2010,2012)$ studies in which people consistently exhibited the south-going route preference heuristic, and it does not account for the increased selections of down-going routes when the routes started in the opposite direction of the destinations in the two-goal condition. We speculate that the different route choice selection instructions promoted different spatial references and caused the contrary results. To be specific, the "choose the better route" instruction promoted an allocentric reference, in which down-going routes were preferred, whereas the "choose the route you prefer to use" instruction caused the participants to imagine they were standing at the origin and, therefore, promoted an egocentric reference, in which up-going routes were preferred. This explanation provides a good account of the results of prior studies (Brunyé et al., 2010, 2012), in which real-world maps were used and the participants were asked to select the "best route." In this case, a geographic reference was probably promoted and southern routes were preferred. Moreover, this explanation accounts for the results of the two-goal maps. In the two-goal condition, the participants probably imagined themselves standing at the origin. Thus, the directions of the up-going routes aligned with their head directions. However, when the initial segments of the up-going routes were in the opposite direction of the egocentric head directions (in the start-against-goal condition), the preference for the up-going routes disappeared. To provide additional support for this hypothesis, we conducted the second experiment, in which the route choice instructions were changed and geographic compasses were included.

\section{Experiment 2}

In Experiment 1, a down-going route preference heuristic was observed when the participants were asked to select the "better route," but contrary preferences appeared when the participants were asked to select "the route they prefer to use." The instruction of selecting the better (or the best) route may promote an allocentric reference which favors a down-going route preference. If an egocentric reference is promoted (such as the case in the two-goal condition), up-going routes in which route directions align to head direction should be preferred. In Experiment 2, the route choice instruction was changed to "select the route you think is easier to remember and easier to travel." This instruction was expected to promote an egocentric reference and induce an up-going route preference. Furthermore, we presented direction compasses together with the maps, as described in previous studies (e.g., Brunyé et al., 2010, 2012), to confirm the dominance of the egocentric reference. That is, if the participants ignored the directions shown by the compasses, the egocentric reference is considered dominant. Finally, we hypothesized that the "choose the route you prefer to use" instruction used in the two-goal condition would promote an

Table 1.

Mean percentages and standard deviations of down-going and right-going route selections in each condition of Experiment 1.

\begin{tabular}{ccccc}
\hline & \multicolumn{2}{c}{ Up-down maps } & \multicolumn{2}{c}{ Left-right maps } \\
\hline & Start-toward-goal & Start-against-goal & Start-toward-goal & Start-against-goal \\
Two-start & $.63(.31)^{* *}$ & $.61(.29)^{* *}$ & $.53(.30)$ & $.55(.33)$ \\
Two-goal & $.36(.31)^{* *}$ & $.56(.34)$ & $.58(.32)$ & $.49(.29)$ \\
\hline
\end{tabular}

Note: For each condition, the participants' selections were compared to chance level. ${ }^{* *} p<.01$. 
egocentric reference. However, the two-destination condition may also have influenced spatial reference. In order to exclude this possibility, we only used two-start maps and compared the results with those of Experiment 1.

\section{Method}

\section{Participants}

Ninety-eight participants ( 80 females, average age $=19.1$ years, six left-handers) completed the route choice questionnaire. None of these individuals participated in Experiment 1.

\section{Materials}

Compasses showing north were added to the two-start maps used in Experiment 1. The compasses were illustrated as a triangle within a circle; the word "north" was printed near the acute angle of the triangle (Figure 2). The compasses indicated north as any direction (up, down, left, or right) in each map. The routes were curved or had right angles. Half of the maps contained up-down routes, and the others contained left-right routes. Half of the routes started toward the destinations, and the others started in the direction opposite the destination. A total of 32 maps were included in the questionnaire (2 map types, 4 compasses, 2 start directions, 2 shapes). The maps were printed in random order.

\section{Procedure}

Prior to completing the root choice task, the participants completed the Santa Barbara Sense of Direction (SBSOD) scale (Hegarty, Richardson, Montello, Lovelace, \& Subbiah, 2002). Next, for each map, they read the explanation of the map and the route choice task, and were asked to select the route that they thought was easier to remember and find. As in Experiment 1 , they were instructed to make intuitive decisions and not to change their prior choices.

\section{Results}

For all up-down maps, the percentage of down-going routes selected is shown in Table 2. Similarly, for all left-right maps, the percentage of right-going routes selected is also shown in Table 2. The percentage of down-going and right-going selections was compared to chance level $(50 \%)$ after the application of angular transformations. For up-down maps, the selections of down-going routes were significantly less than chance level when the initial segments were toward the destinations $(t(97)=$ $-6.70, p<.01)$, but at chance level when the routes started against the destinations $(t(97)=-1.80$, n.s. $)$. For left-right maps, right-going routes were preferred in both the start-toward-goal condition and the start-against-goal condition $(t(97)=2.73, p$ $<.01 ; t(97)=4.12, p<.01$, respectively).

Furthermore, the main effect of compasses was significant for both up-down and left-right maps $(F(3,291)=7.41, p<.01$;
$F(3,291)=4.46, p<.01$, respectively). For the up-down maps, there were significantly more selections of down-going routes when the compasses pointed down (i.e., north was down) than the other conditions (Tukey's Honestly Significant Difference (HSD) tests, $p s<.05$ ). For the left-right maps, the selections of right-going routes were greater than chance level when the compasses pointed to the right, up, and down, but were at chance level when the compasses pointed to the left $(t(97)=$ $4.81, p<.01 ; t(97)=2.98, p<.01 ; t(97)=2.13, p<.05 ; t(97)=$ 0.70 , n.s., respectively). We also statistically analyzed the geographic route choices (Table 3) (i.e., a south-going route refers to a down-going route when the compass pointed up, an upgoing route when the compass pointed down, a right-going route when the compass pointed left, and a left-going route when the compass pointed to the right) and found significant differences in geographic route choices $(F(3,291)=10.82, p$ $<.01)$. There were fewer selections of the south-going routes than the other route directions (Tukey's HSD tests: $p \mathrm{~s}<.01$ ). Finally, the mean SBSOD score for all participants was 3.28 (7-point scale, $S D=1.10)$. The mean score was not signifi-
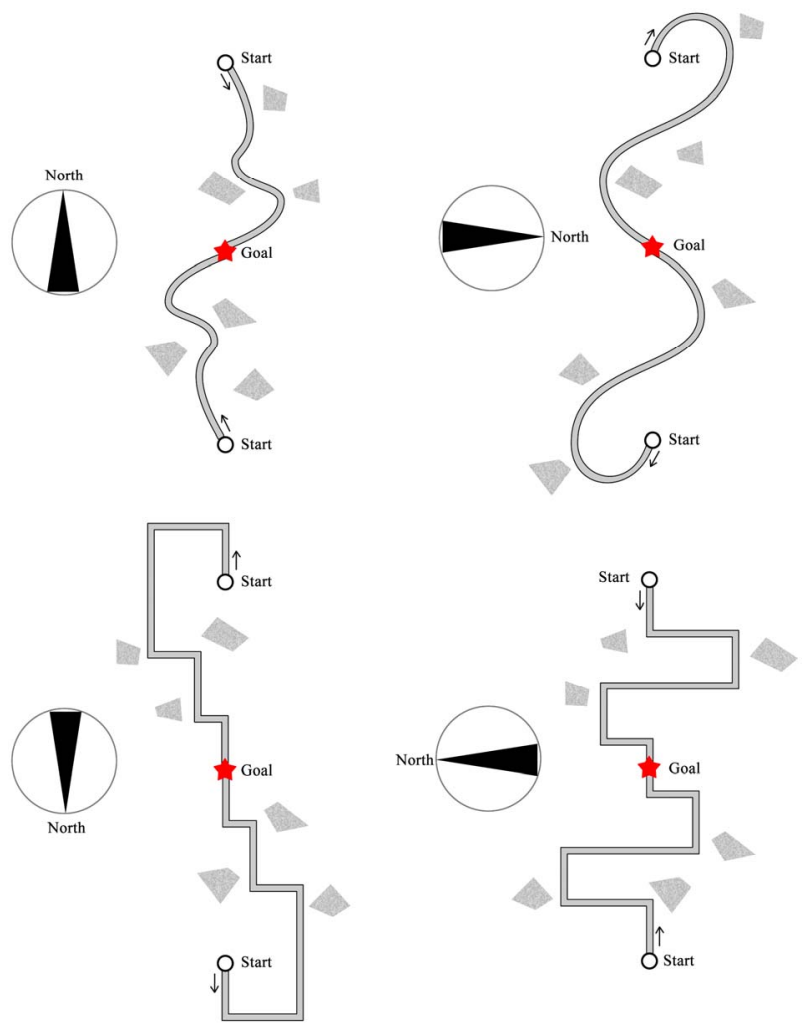

Figure 2.

Map examples showed to the participants. Compasses showing north were added to the map.

Table 2.

Mean percentages and standard deviations of down-going and right-going route selections in each condition of Experiment 2.

\begin{tabular}{cccc}
\hline \multicolumn{2}{c}{ Up-down maps } & \multicolumn{2}{c}{ Left-right maps } \\
\hline Start-toward-goal & Start-against-goal & Start-toward-goal & Start-against-goal \\
$.33(.23)^{* *}$ & $.46(.23)$ & $.57(.21)^{* *}$ & $.59(.21)^{* *}$ \\
\hline
\end{tabular}

Note: For each condition, the participants' selections were compared to chance level. ${ }^{* *} p<.01$. 
Table 3.

Mean percentages and standard deviations of geographic route choice.

\begin{tabular}{cccc}
\hline South-going & North-going & East-going & West-going \\
\hline $.21(.10)$ & $.29(.10)$ & $.25(.06)$ & $.25(.06)$ \\
\hline
\end{tabular}

cantly correlated with down-going route preference, right-going route preference, or any of the geographic route choices, showing that the route preferences were not related to sense of direction.

\section{Discussion}

Contrary to the down-going route preference in Experiment 1, the participants in this experiment preferred up-going routes when selecting between two routes starting from different origins to the same destination. For Experiment 2, the route choice instruction was changed from "select the better route" to "select the route you think is easier to remember and easier to find." As expected, the latter instruction promoted an egocentric reference and induced an up-going route preference. This hypothesis was supported by the results of the start-against-goal condition. That is, the up-going route preference disappeared when the initial segments of the routes were inconsistent with the egocentric head directions.

Furthermore, the analysis of geographic route choice showed a negative priming effect of compasses. Routes misaligning to the compass were avoided; this finding indicates that this perceptual feature on maps could also influence route choices. This interpretation has important implications for map design and needs further discussion. However, another explanation of the geographic route choices results is that the participants simply did not want to go south. Unfortunately, this hypothesis is inconsistent with the findings of Brunyé and colleagues (2010, 2012) and of Experiment 1 in the present study. Finally, the observed right-going route preference in the left-right condition was probably a result of a high proportion of right-handers.

In Experiments 1 and 2, we found that people favored a down-going route when an allocentric reference was dominant but preferred an up-going route when an egocentric reference was dominant. Brunyé and others $(2010,2012)$ suggested that the down-going direction may be associated with the vertically down direction and is perceived to be less physically demanding. Although this is possible, it does not account for the fact that spatial references changed route preference.

\section{Experiment 3}

It is still not clear how this heuristic of different route preferences in an allocentric and egocentric reference profits spatial behaviors. Therefore, in Experiment 3, we asked people to memorize a down-going or an up-going route. We then examined their wayfinding and spatial memory performance.

\section{Method}

\section{Participants}

Twenty-six individuals ( 12 females, mean age $=24.5$ years $)$ were recruited. None of them participated in Experiments 1 or 2 or entered the building used for wayfinding prior to the experiment.

\section{Materials}

We planned two routes (Figure 3) from the Faculty of Law
\& Letters Building 2 of the University of Tokyo, which has five floors, an L shape, a quadrangle, and six exits. The two routes were $83 \mathrm{~m}$ and $78 \mathrm{~m}$ in length and had five and six turns, respectively. For each route, four landmarks were specified and printed on the map as icons. The two routes did not meet or cross with each other, and were used for either an up-going route or a down-going route, by rotating the route $180^{\circ}$. Photos near landmarks and goals were presented to the participants.

\section{Procedure}

Participants took part in the experiment individually. They first completed the SBSOD scale (Hegarty et al., 2002) outside the building, and then were told that they were going to memorize a map, find the goal without using the map, and draw a sketch-map after wayfinding. They were told not to rotate the map and to find the goal as quickly as possible. Then, they studied the first map until they reported that they had memorized the whole map. After map learning, the participants followed the experimenter to the start place, and were shown the start direction. During the wayfinding, if the participants deviated from the route for more than $5 \mathrm{~s}$, they were redirected to the correct route by the experimenters. The total time, number of errors, and number of stops was recorded. After arriving at the goal, the participants returned to the place where they had received an explanation of the experiment and drew a sketchmap on a blank piece of A4-sized $(210 \times 297 \mathrm{~mm})$ paper. After the first trial, the participants rested for about five minutes. Next, they memorized the second map, and completed the wayfinding and the map-sketching tasks. After finishing all the tasks, they gave an oral report of their navigating strategies and map-reading habits used in their daily life. The order and orientation of routes were counter-balanced between participants. The experiment lasted $\sim 40$ minutes for each participant.

\section{Results}

We excluded data from two participants, because one rotated the map during memorizing phase and another failed the wayfinding task. For the wayfinding task, the total time, number of errors, and number of stops were used to index task performance (Table 4). For the map-sketching task, the proportion of correct turns and the correlation of bidimensional regression

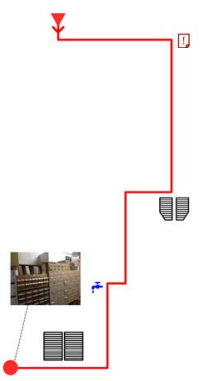

(A)

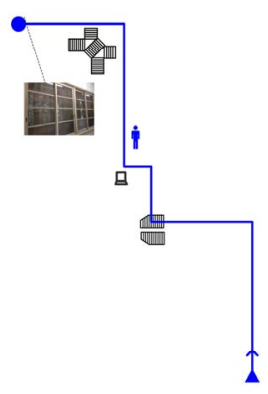

(B)
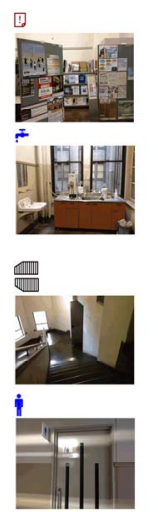

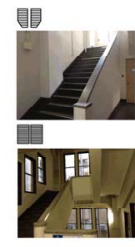

日

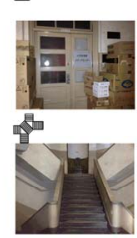

Figure 3.

The routes used in Experiment 3. The two routes served as either a down-going route or an up-going route. Photos near the goals and icons of landmarks were printed on the maps. Photos taken near the landmarks were printed on a separate piece of paper. 
(Tobler, 1965) were used to examine the participants' route knowledge and configurational knowledge, respectively (Table 4). In the bidimensional regression, start and end points and the four landmarks were used as "anchors." A Fisher's $r$-to- $z$ transformation was applied to the bidimensional correlations for analysis.

The differences in total time to complete the wayfinding task and the correlations of the map-sketching task were marginally significant $(t(21)=1.93, p=.07 ; t(21)=-1.98, p=.06$, respectively). The participants found goals faster but sketched positions of landmarks less accurately when they learned an upgoing route. There were no significant differences in the other indices of task performance. Furthermore, the average SBSOD score of the participants was $4.41(S D=1.44)$ and was significantly related to only the number of stops when down-going routes were used $(r=-.45, p<.05)$.

\section{Discussion}

The results of Experiment 3 indicated that the participants acquired better egocentric route knowledge from the up-going routes, and acquired better allocentric configurational knowledge from the down-going routes. In the up-going routes condition, the orientation of the routes was aligned to the participant's head direction. Thus, turning directions (left or right) were easy to recall during wayfinding. However, in the downgoing route condition, to decide whether to turn left or right, the participants had to mentally rotate the memorized route, which resulted in longer wayfinding times. Moreover, the negative correlation between SBSOD score and the number of stops in the down-going route condition reflected the demands of the mental rotations. On the other hand, the down-going route probably facilitated configurational knowledge by promoting an allocentric reference. In the up-going routes condition, the participants might have associated the positions of landmarks with the routes (e.g., there were stairs after turning left). However, in the down-going routes condition, route knowledge occurred at a greater cost, and the participants probably encoded positions of landmarks directly with an allocentric reference (e.g., out of environmental frames or the shape of the entire route), which resulted in more accurate configurational knowledge.

\section{General Discussion}

From the results of Experiments 1 and 2, we discovered that people did not always choose the route they thought was easier to remember and easier to go for the "better route." Although these results are counterintuitive, they reflected the relation

\section{Table 4.}

The total time, number of errors, and number of stops in the wayfinding task. The proportion of correct turns and the bidimensional correlation in the map-sketching task.

\begin{tabular}{cccc}
\hline & & Down-going route & Up-going route \\
\hline \multirow{3}{*}{ Wayfinding task } & Total time & 91 seconds & 83 seconds \\
& Errors & 0.5 & 0.8 \\
& Stops & 1.0 & 1.1 \\
\multirow{2}{*}{$\begin{array}{c}\text { Map-sketching } \\
\text { task }\end{array}$} & Correct turns & $97 \%$ & $99 \%$ \\
\hline
\end{tabular}

between route choices and selections on spatial references, which was ignored by most of the prior studies on route choice. As a consequence of being asked to select the "best/better route," an allocentric reference was promoted and down-going routes were favored, as was reported by Brunyé et al. (2010, 2012). However, as a result of being asked to select "the route you want to go much more" or "the route that seems to be easier to remember and easier to find," an egocentric reference dominated and up-going routes were preferred. The results demonstrated that different route choice instructions may influence the dominance of spatial reference and reserve route preferences. This fact should be acknowledged in further research of route choice.

Furthermore, the results of Experiment 3 provided a behavioral rationale for the abovementioned heuristics of route preference. As a result of being asked to select the best route, people paid more attention to the global spatial layout, which facilitated configurational spatial knowledge and lead to a downgoing route preference. In contrast, although the participants indicated that they would actually use the route later, they probably paid more attention to the turning directions of the routes, which were associated with egocentric route knowledge and resulted in an up-going route preference.

Although the heuristics of route preference may sometimes be inefficient, they reflect a person's navigating strategies and spatial cognitive processes. The present study started from an interest in the heuristics of south-going (down-going) route preference as reported by prior studies (Brunyé et al., 2010, 2012), and found important relations between down-going and upgoing route preferences and spatial references. In conclusion, when selecting the "best route," people pay attention to configurational information and prefer a down-going route. In contrast, when selecting "the way easier to travel," people turn to egocentric route information and favor an up-going route. The present study is the first to clarify the relation between route preferences and spatial references, and provides important knowledge about route choice instructions to be used in future studies.

\section{Acknowledgements}

We thank Prof. Toru Ishikawa for valuable research advice. We also thank Prof. Takao Sato for supporting these experiments.

\section{REFERENCES}

Bailenson, J. N., Shum, M. S., \& Uttal, D. H. (1998). Road climbing: Principles governing asymmetric route choices on maps. Journal of Environmental Psychology, 18, 251-264. doi:10.1006/jevp.1998.0095

Bailenson, J. N., Shum, M. S., \& Uttal, D. H. (2000). The initial segment strategy: A heuristic for route selection. Memory \& Cognition, 28, 306-318. doi:10.3758/BF03213808

Brunyé, T. T., Andonova, E., Meneghetti, C., Noordzij, M. L., Pazzaglia, F., Wienemann, R., Mahoney, C. R. et al. (2012). Planning routes around the world: International evidence for southern route preferences. Journal of Environmental Psychology, 32, 297-304. doi:10.1016/j.jenvp.2012.05.003

Brunyé, T. T., Mahoney, C. R., Gardony, A. L., \& Taylor, H. A. (2010). North is up(hill): Route planning heuristics in real-world environments. Memory \& Cognition, 38, 700-712. doi:10.3758/MC.38.6.700

Christenfeld, N. (1995). Choice from identical options. Psychological Science, 6, 50-55. doi:10.1111/j.1467-9280.1995.tb00304.x 


\section{W. WEN, H. KAWABATA}

Hegarty, M., Richardson, A. E., Montello, D. R., Lovelace, K., \& Subbiah, I. (2002). Development of a self-report measure of environmental spatial ability. Intelligence, 30, 425-447.

doi:10.1016/S0160-2896(02)00116-2

Hochmair, H. H., \& Karlsson, V. (2005). Investigation of preference between the least-angle strategy and the initial segment strategy for route. Lecture Notes in Computer Science, 3343, 79-97. doi:10.1007/978-3-540-32255-9 5

Tobler, W. R. (1965). Comparison of the correspondence of geographic patterns. Papers and Proceedings of the Regional Science Association, 15, 131-139. doi:10.1007/BF01947869

Yang, J., \& Schwaninger, A. (2011). Turn right or turn left? Heuristic of adhering to the direction of destination. Applied Cognitive Psychology, 25, 703-707. doi:10.1002/acp.1741 